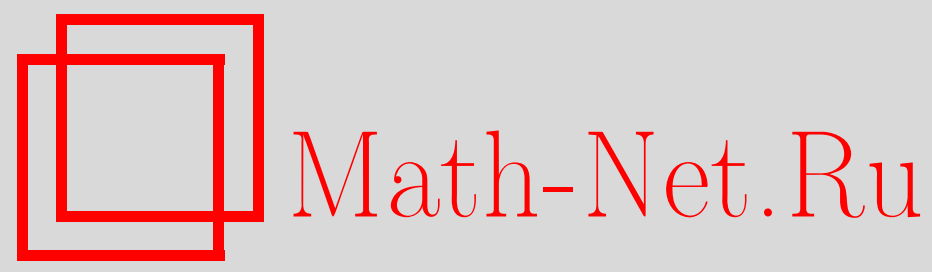

И. С. Лапшов, Динамические базы данных с оптимальной по порядку временной сложностью, Дискрет. матем., 2008, том 20, выпуск 3, 89-100

DOI: https://doi.org/10.4213/dm1016

Использование Общероссийского математического портала Math-Net.Ru подразумевает, что вы прочитали и согласны с пользовательским соглашением http://www . mathnet.ru/rus/agreement

Параметры загрузки:

IP : 3.81 .55 .215

26 апреля 2023 г., 14:44:56 


\title{
Динамические базы данных с оптимальной по порядку временной сложностью
}

\author{
() 2008 г. . И. С. Лапшов
}

\begin{abstract}
В работе исследуется сложность таких основных операций в динамических базах данных, как поиск, вставка и удаление записей. Предложены структура базы данных и соответствующие ей алгоритмы, которые с помощью двух параллельных вычислительных процессов позволяют совершать основные операции над базой данных в среднем за константное время, в то время как для худшего случая справедлива логарифмическая оценка сложности.
\end{abstract}

\section{1. Введение}

Объектом исследования являются динамические базы данных, то есть структуры, хранящие информацию и предоставляющие пользователю набор операций для поиска и обработки этой информации.

В $[1,2]$ была рассмотрена модель динамической базы данных, выполняющей запросы на поиск, вставку и удаление записей в среднем за константное время. В качестве представления динамической базы данных (БД) использовалась информационнографовая модель данных [3]. В этой модели состоянию базы данных в каждый момент времени сопоставляется ориентированный граф с нагруженными ребрами и вершинами. Основным операциям, поиску, вставке и удалению записей из БД, соответствовали процедуры, выполняющие перемещения по ребрам графа и модифицирующие его структуру. Исследовалась сложность основных операций как время выполнения соответствующих процедур в среднем и в худшем случаях. Было показано, что в построенной модели средняя сложность этих операций не превосходит константу. Небольшая средняя сложность достигалась за счет введения дополнительного, служебного вычислительного процесса, поддерживающего структуру базы данных по мере ее наполнения. Однако, существенным недостатком такой модели является линейная относительно числа записей сложность в худшем случае основных операций.

В данной работе предлагается алгоритм основных операций, который в среднем эффективен так же, как алгоритм, описанный в [1]. Однако, сложность в худшем случае основных операций имеет логарифмический порядок, такой же как у большинства древовидных структур, использующихся для реализации динамических баз данных.

Автор благодарит Э. Э. Гасанова за постановку задачи и помощь в работе. 


\section{2. Основные понятия и формализация модели}

Для исследования необходимо построить формальную модель.

Назовем некоторое множество $Y$ множеством записей, а элементы этого множества записями. Будем считать, что информация, хранящаяся в базе данных, представляет собой конечное подмножество $V$ множества записей. Назовем это подмножество библиотекой.

Будем рассматривать три вида операций над базами данных: поиск записи, вставка записи и удаление записи.

Для выполнения поиска необходим поисковый запрос - объект из множества $X$, которое мы назовем множеством поисковых запросов. В теории поиска информации вводится также бинарное отношение $\rho \subseteq X \times Y$, называемое отношением поиска. Запись $y \in Y$ удовлетворяет запросу $x \in X$, если $x \rho y$. Тройку $\langle X, Y, \rho\rangle$ называют типом поиска. Операция поиска по запросу $x \in X$ состоит в отыскании всех записей $y \in V$ таких, что $x \rho y$.

Далее будем рассматривать так называемый поиск идентичных объектов, то есть поиск типа $\langle X, Y, \rho\rangle$, где $X=Y$, а $\rho$ - отношение равенства на $Y \times Y$. В качестве модели базы данных будем использовать информационные графы (ИГ).

Структура ИГ. Пусть $F-$ множество одноместных предикатов, заданных на множестве $X$. Пусть $G$ - множество функций, определенных на $X$, области значений которых являются отрезками натурального ряда. Назовем их переключателями. Пару $\mathscr{F}=\langle F, G\rangle$ будем называть базовым множеством.

Пусть нам дан произвольный ориентированный граф (возможно, пустой). Выделим в нем одну вершину (если граф непустой) и назовем ее корнем ИГ. Также выделим произвольное подмножество вершин графа и назовем их листьями (корень может быть листом). Вершины графа, не являющиеся листьями, будем называть внутренними вершинами. Сопоставим каждому листу графа некоторую запись из множества $Y$. Это соответствие назовем нагрузкой листьев.

Выделим некоторое подмножество внутренних вершин, которые назовем точками переключения. Каждой точке переключения $v$ с полустепенью исхода $m$ поставим в соответствие переключатель $g_{v}(x) \in G$, у которого область значений содержит не менее $m$ чисел. Занумеруем все дуги, исходящие из $v$ последовательными числами из области значений $g_{v}$. Такие дуги назовем переключательными. Внутренние вершины, не являющиеся точками переключения, назовем предикатными. Дуги, не являющиеся переключательными, также назовем предикатными. Каждой предикатной дуге сопоставим символ из $F$.

Полученный граф назовем информационным графом над базовым множеством $\mathscr{F}$ с библиотекой $V$, где $V$ - множество записей, приписанных листьям ИГ.

Поиск в ИГ. Каждому ИГ $U$ можно сопоставить процедуру поиска. Предполагается, что эта процедура хранит в своей (внешней) памяти структуру $U$. Входными данными является запрос $x \in X$. Выходными данными является подмножество библиотеки $V$.

Пусть на вход процедуре поступил запрос $x \in X$. Если ИГ пустой, то процедура поиска завершается с пустым выходным множеством. В противном случае введем понятие активного множества вершин и внесем в него в начальный момент корень ИГ $U$ и помечаем его. Далее по очереди просматриваем вершины из активного множества и для каждой из них проделываем следующее:

- если рассматриваемая вершина - лист, то запись, приписанную вершине включаем в ответ; 
- если рассматриваемая вершина - точка переключения, то вычисляем на запросе $x$ переключатель, соответствующий данной вершине, и если дуга, нагрузка которой равна значению переключателя существует и ее конец - непомеченная вершина, то помечаем конец дуги и включаем его в множество активных вершин;

- если рассматриваемая вершина предикатная, то просматриваем по очереди исходящие из нее дуги и вычисляем значения предикатов, приписанных этим дугам на запросе $x$. Концы дуг, которым соответствуют предикаты со значениями, равными 1, если они непомеченные, помечаем и включаем в множество активных вершин;

- исключаем рассматриваемую вершину из активного множества.

Процедура завершается по исчерпании активного множества.

Скажем, что ИГ $U$ решает задачу поиска, если множество, полученное на выходе процедуры, будет содержать все те и только те записи библиотеки $V$, которые удовлетворяют запросу $x$.

Сложность поиска в ИГ. Пусть нам дан ИГ $U$ над базовым множеством $\mathscr{F}$. Каждому символу $g \in G(f \in F)$ поставим в соответствие неотрицательное число $t(g)(t(f))-$ сложность переключателя $g$ (предиката $f$ ). Тогда понятие базового множества функций можно расширить, рассматривая функции из $F$ и $G$ вместе со своими сложностями. Будем записывать это как $\langle F, G, t\rangle$

Сложностью поиска по запросу $x$ в ИГ $U$ назовем сумму сложностей всех вычисленных в ходе процедуры поиска переключателей и предикатов. Обозначим сложность поиска $T(U, x)$.

Сложность ИГ $U$ можно вводить двумя способами. Во-первых, как максимальную сложность на запросе

$$
\widehat{T}(U)=\max _{x \in X} T(U, x) .
$$

Эту величину будем называть верхней сложностью ИГ $U$, или сложностью в худшем случае.

Во-вторых, можно вводить среднюю сложность ИГ $U$. Для этого будем считать, что $x$ - случайная величина с неким заданным вероятностным распределением на множестве $X$. Тогда средняя сложность поиска в ИГ $U$ будет определяться как математическое ожидание величины $T(U, x)$, то есть как число

$$
T(U)=\mathbf{M}_{x} T(U, x)
$$

Вставка и удаление записи в ИГ. Назовем вставкой записи $y$ в ИГ $U$ такое преобразование $U$, при котором библиотека $V$, приписанная листьям ИГ $U$, преобразуется в $V \cup\{y\}$ и $U$ остается ИГ, решающим задачу поиска для библиотеки $V \cup\{y\}$.

Удалением записи $y$ из ИГ $U$ назовем преобразование $U$, при котором библиотека $V$, приписанная листьям ИГ $U$, преобразуется в $V \backslash\{y\}$ и $U$ остается ИГ, решающим задачу поиска для библиотеки $V \backslash\{y\}$.

Будем рассматривать вставку (удаление) записи в ИГ как процедуру, хранящуюся во внешней памяти структуры ИГ и выполняющуюся по некому условному алгоритму $A$. На вход процедуре подается элемент $y \in Y$, а результатом ее работы является преобразование $U$.

Алгоритм вставки/удаления представляет собой последовательность преобразований вершин, дуг графа, их меток, которые мы договоримся считать элементарными, то есть на 
выполнение которых тратится небольшое и константное время. Набор конкретных преобразований будем называть базовым множеством преобразований и обозначать $\mathscr{H}=\langle H\rangle$, где $H=\left\{h_{1}, \ldots h_{m}\right\}-$ множество элементарных операций.

Пусть дан пустой ИГ. Осуществим некоторое число последовательных вставок и удалений записей $y_{1}, \ldots, y_{l}, l \in \mathbf{N}_{0}$. Полученный ИГ назовем построенным по набору $\left(y_{1}, \ldots, y_{l}\right)$. Обозначим через $U(A, n)$ множество ИГ с $n$ листьями, построенных по алгоритму $A$ по всевозможным наборам из $Y$. Введем также обозначение

$$
u(A)=\bigcup_{n \in \mathbf{N}} u(A, n) .
$$

Вставка/удаление записи $y \in Y$ в ИГ $U$ по алгоритму $A$ имеет смысл, только если $U \in \mathcal{U}(A)$.

Сложность вставки/удаления записи в ИГ. Сопоставим каждому $h \in H$ величину $t(h) \geqslant 0$ и назовем ее сложностью элементарной операции $h$. Значения таких величин будем обговаривать отдельно. Тогда можно расширить понятие базового множества элементарных операций, рассматривая каждую операцию вместе со своей сложностью. Обозначим это символом $\mathscr{H}=\langle H, t\rangle$.

Любой алгоритм $A$ вставки/удаления строится над некоторым базовым множеством функций $\mathscr{F}=\langle F, G, t\rangle$ и базовым множеством преобразований $\mathscr{H}=\langle H, t\rangle$. Сложность вставки записи $y \in Y$ в ИГ $U$ по алгоритму $A$ будет равна сумме сложностей элементарных операций, произведенных в ходе выполнения процедуры по этому алгоритму. Обозначим ее $R_{A}(U, y)$. Аналогично, сложность удаления записи определим как сумму сложностей соответствующих элементарных операций и обозначим еe $S_{A}(U, y)$. Величину

$$
\hat{R}_{A}(U)=\max _{y \in Y} R_{A}(U, y)
$$

назовем верхней сложностью (или сложностью в худшем случае) вставки в ИГ $U$. Величину

$$
\widehat{S}_{A}(U)=\max _{y \in Y} S_{A}(U, y)
$$

назовем верхней сложностью (или сложностью в худшем случае) удаления в ИГ $U$.

Считая $y$ случайной величиной, среднюю сложность вставки записи $y$ в ИГ $U$ можно определять как

$$
R_{A}(U)=\mathbf{M}_{y} R_{A}(U, y) .
$$

Аналогично, среднюю сложность удаления записи $y$ из ИГ $U$ определим как

$$
S_{A}(U)=\mathbf{M}_{y} S_{A}(U, y) .
$$

Объемом $Q(U)$ ИГ $U$ назовем число ребер в ИГ $U$. Число листьев в ИГ $U$ обозначим $N(U)=|V|$, где $V-$ библиотека ИГ $U$.

\section{3. Исследование 2-3 деревьев}

Предлагаемая модель функционирования динамической базы данных использует понятие 2-3 дерева. Именно за счет применения 2-3 деревьев время выполнения основных операций имеет порядок логарифма от числа записей в библиотеке. Опишем структуру 2-3 дерева. 


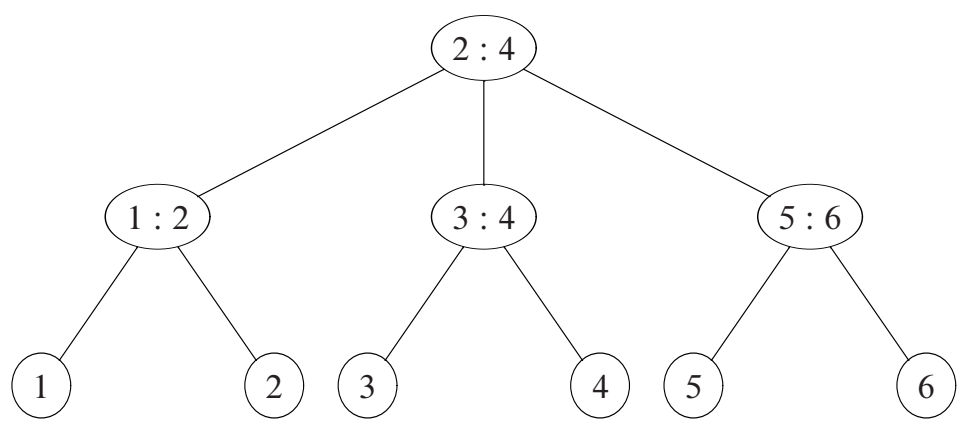

Рис. 1. Пример 2-3 дерева

2-3 деревом называется дерево, в котором каждая вершина, не являющаяся листом, имеет двух или трех сыновей, а длины всех путей из корня в листья одинаковы (см. гл. 4.9 в [4]). Заметим, что дерево, состоящее из единственного узла, является 2-3 деревом. Пример 2-3 дерева дан на рис. 1.

Линейно упорядоченное множество (в нашем случае - числа $\{1, \ldots, n\}$ можно представить с помощью 2-3 дерева следующим образом: элементы множества приписываются листьям слева направо по порядку. В каждой внутренней вершине $v$ хранятся две величины: $L[v]$ - максимальный элемент в левом поддереве узла $v$ и $M[v]-$ максимальный элемент в среднем (если две дуги - то в правом) поддереве $v$.

Таким образом, операция поиска элемента $y$ в 2-3 дереве происходит путем последовательного сравнения по следующему алгоритму: если $y<L[v]$, то переходим к левому сыну $v$, в противном случае, если у $v$ два сына, или $v<M[v]$, то переходим к среднему сыну $v$, в противном случае переходим к правому сыну $v$.

Чтобы в 2-3 дерево вставить новый элемент $y$, надо найти место для нового листа $l$, который будет содержать $y$. Для этого ищут элемент $y$ в дереве. Если дерево содержит более одного элемента, то поиск $у$ окончится в узле $f$, имеющим двух или трех сыновей, которые являются листами. Если из узла $f$ выходит только два листа $l_{1}$ и $l_{2}$, то $l$ делаем сыном узла $f$ так, чтобы листья остались упорядоченными слева направо. Кроме того, нужно изменить метки $L$ и $M$ в вершине $f$, а возможно и в нескольких предках $f$.

Теперь предположим, что у $f$ уже есть три листа $l_{1}, l_{2}$ и $l_{3}$. Сделаем $l$ сыном узла $f$, сохраняя упорядоченность листьев. Чтобы сохранить 2-3 свойство, образуем новый узел $g$. Два левых сына оставим сыновьями $f$, а два правых переделаем в сыновей узла $g$. Затем сделаем $g$ братом узла $f$, сделав его сыном отца узла $f$. Назовем эту операцию переброской. Если отец узла $f$ уже имел трех сыновей, то надо рекурсивно повторять переброску до тех пор, пока у всех узлов в дереве останется не более трех сыновей. Если у корня окажется четыре сына, образуем новый корень с двумя новыми сыновьями, каждый из которых будет иметь в качестве двух своих сыновей двух из четырех сыновей старого корня.

Опишем процедуру удаления записи $x$ в 2-3 дереве. Начиная от корня, ищем запись $x$ в дереве. Если запись не найдена, то заканчиваем процедуру удаления. В противном случае пусть $f$ - вершина, имеющая в качестве сына лист, соответствующий записи $x$. Удалим этот лист. Если после удаления у вершины $f$ осталось два сына, то заканчиваем процедуру. Если же у вершины $f$ остался только один сын, то склеим $f$ с любым из ее братьев объединив их листья (заметим, что логично склеивать $f$ с тем из братьев, который 
имеет двух сыновей, если такой брат существует). После этого, возможно, $f$ (точнее новая склеенная вершина) будет единственным сыном своей родительской вершины. В этом случае повторим операцию склейки одним ярусом ближе к корню. Наконец, если после этих операций корень имеет только одного сына, то объявим этого сына новым корнем 2-3 дерева, а старый корень удалим.

Получившееся дерево все еще может не обладать 2-3 свойством, так как при склейке вершин число сыновей может превысить число 3. Поэтому необходим еще один проход от листа (брата удаленного листа) до корня с проверкой числа сыновей у вершин и по необходимости перебросками.

Примем сложность прохождения через внутреннюю вершину равной $t_{\lambda}$, сложность операции переброски равной $t_{2}$, а сложность склейки равной $t_{3}$.

Исследуем сложность поиска, вставки и удаления записи в 2-3 дереве. Обозначим 2-3 дерево, построенное по набору $\bar{y} б$ через $D_{\bar{y}}$. Сложность построения 2-3 дерева по набору $\bar{y}$ обозначим $G(\bar{y})$. Введем величины

$$
\begin{array}{rlrl}
\hat{T}_{23}(n) & =\max _{|\bar{y}|=n} \hat{T}\left(D_{\bar{y}}\right), & \bar{T}_{23}(n)=\mathbf{M}_{\bar{y}} T\left(D_{\bar{y}}\right), & \widehat{R}_{23}(n)=\max _{|\bar{y}|=n} \hat{R}\left(D_{\bar{y}}\right), \\
\bar{R}_{23}(n)=\mathbf{M}_{\bar{y}} R\left(D_{\bar{y}}\right), & \widehat{G}_{23}(n)=\max _{|\bar{y}|=n} G(\bar{y}), & \bar{G}_{23}(n)=\mathbf{M}_{\bar{y}} G(\bar{y}) .
\end{array}
$$

Очевидно следующее утверждение.

Предложение 1. Высота $h$ 2-3 дерева удовлетворяет неравенствам

$$
\left\lceil\log _{3} n\right\rceil \leqslant h \leqslant\left\lfloor\log _{2} n\right\rfloor
$$

где $n$ - число листьев в дереве.

Предложение 2. Справедливы равенства

$$
\begin{aligned}
& \widehat{T}_{23}(n)=t_{\lambda}\left\lfloor\log _{2} n\right\rfloor, \\
& \widehat{R}_{23}(n)=\left(t_{\lambda}+t_{2}\right)\left\lfloor\log _{2} n\right\rfloor, \\
& \widehat{S}_{23}(n)=\left(t_{\lambda}+t_{2}+t_{3}\right)\left\lfloor\log _{2} n\right\rfloor .
\end{aligned}
$$

Доказательство. Поиск записи $x$ в 2-3 дереве $D_{23}$ осуществляется путем прохождения от корня до соответствующего листа (или вершины предпоследнего яруса в случае, если записи $x$ нет в библиотеке). По определению, длины всех таких путей равны. Используя предложение 1 о высоте 2-3 дерева, получим искомое неравенство $\widehat{T}_{23}=t_{\lambda}\left\lfloor\log _{2} n\right\rfloor$.

Вставка записи $x$ в дерево включает в себя поиск $x$ в дереве, а затем, возможно, несколько перебросок, число которых также не превышает высоту дерева. Поэтому $\widehat{R}_{23}=\left(t_{\lambda}+t_{2}\right)\left\lfloor\log _{2} n\right\rfloor$.

Удаление записи $x$ в 2-3 дереве также состоит из поиска $x$ в дереве, затем, возможно, нескольких склеек и перебросок (в обоих случаях их число не превышает высоту дерева). Получаем, что $\widehat{S}_{23}=\left(t_{\lambda}+t_{2}+t_{3}\right)\left\lfloor\log _{2} n\right\rfloor$.

\section{Хеширование с использованием 2-3 деревьев}

В [1] была описана структура БД с константной в среднем сложностью основных операций. Однако, как отмечалось выше, построенная модель обладает одним недостатком большим порядком сложности в худшем случае. 
Ниже будет показано, что, используя структуры с логарифмической сложностью основных операций и 2-3 деревья, можно добиться того, что верхняя сложность этих операций будет порядка $\log n$, где $n-$ число записей в БД.

Вначале введем новый тип операции над 2-3 деревом - операцию деления пополам. Пусть все записи в дереве лежат на полуотрезке $(a, b]$. Суть операции деления пополам заключается в преобразовании 2-3 дерева $D_{23}$ в два 2-3 дерева $D_{23}^{(1)}$ и $D_{23}^{(2)}$ таких, что все записи в дереве $D_{23}^{(1)}$ лежат на полуотрезке $(a,(a+b) / 2]$, а все записи в дереве $D_{23}^{(2)}-$ на полуотрезке $((a+b) / 2, b]$. Объединение же множеств записей в полученных деревьях должно образовывать множество записей в исходном дереве.

Опишем алгоритм деления. Пусть $d=(a+b) / 2$.

(1) Найдем в дереве вершину $v_{0}$ такую, что в каждом из ее поддеревьев либо все записи меньше или равны $d$, либо все записи больше $d$. Для этого вначале будем перемещаться по дереву от корня к листьям, в каждой вершине $v$ сравнивая значение $d$ со значениями $L[v]$ и $M[v]$. Если вершина $v$ - лист, то переходим к родителю текущей вершины и выполняем шаг 2. Если $d=L[v]$ или $d=M[v]$, то переходим к шагу 2. В противном случае переходим к дочерней вершине так, как если бы мы искали $d$ в 2-3 дереве и повторяем шаг 1 .

(2) Мы пришли в вершину $v_{0}$ такую, что для каждого ее поддерева известно, что либо все записи поддерева меньше или равны $d$, либо все записи больше $d$. Если все поддеревья в этом смысле одного типа, то переходим к родительской вершине (в случае, если $v_{0}$ - корень дерева, то заканчиваем процедуру) и для нее повторяем шаг 2. В противном случае переходим к шагу 3.

(3) Создаем новую вершину $v_{1}$ и делаем ее братом $v_{0}$ (то есть сыном родителя $v_{0}$ ). Все поддеревья, значения в которых больше, чем $d$, делаем сыновьями $v_{1}$. Переходим к родителю $v_{0}$ и повторяем это расщепление для него (мы опять знаем, по какой границе расщеплять поддеревья - между $v_{0}$ и $v_{1}$ ). Продолжаем это расщепление пока не дойдем до корня. По тому же принципу расщепляем корень. Переходим к шагу 4.

(4) Таким образом мы получим два дерева, вообще говоря, не обладающие 2-3 свойством. Чтобы восстановить это свойство, как и в случае удаления, сделаем два прохода в каждом из полученных деревьев: от вершин $v_{0}$ (соответственно, $v_{1}$ ), склеивая вершины, имеющие одного потомка, затем по тому же пути, совершая переброски.

Пусть каждая операция расщепления, совершаемая на 3-м шаге, имеет сложность $t_{4}$. Сложность деления дерева $D_{23}$ пополам обозначим $F\left(D_{23}\right)$.

Предложение 3. Для любого 2-3 дерева $D_{23}$ с $n$ листьями

$$
F\left(D_{23}\right) \leqslant\left(t_{\lambda}+2 t_{2}+2 t_{3}+t_{4}\right)\left\lfloor\log _{2} n\right\rfloor .
$$

Доказательство. Действительно, для деления $D_{23}$ пополам вначале совершается один проход в направлении от корня к листьям по правилам поиска. Сложность этого прохода будет не больше $t_{\lambda}\left\lfloor\log _{2} n\right\rfloor$. Затем производится серия расщеплений, число которых не может превышать высоты дерева, его сложность не больше $t_{4}\left\lfloor\log _{2} n\right\rfloor$. Наконец, для каждого из двух получившихся деревьев производятся серии склеек и перебросок, их сложность не более $2\left(t_{2}+t_{3}\right)\left\lfloor\log _{2} n\right\rfloor$. Просуммировав полученные величины, получим доказываемое неравенство. 
Перейдем к определению структуры, рассматриваемой в данной работе.

Пусть множество $X$ имеет вид $(0,1]$. Рассмотрим переменную $m$ и разбиение $X$ на $m$ полуотрезков. Построим ИГ $U$ над произвольной библиотекой $V \subseteq X$.

Создадим вершину $\beta_{0}$ и объявим ее вершиной графа. Припишем корню переключатель $g_{m}(x)$, где $g_{m}(x)=\lceil x m\rceil$. Пусть $t\left(g_{m}\right)=t_{g}$ для всех $m \in \mathbf{N}$. Из $\beta_{0}$ выпустим $m$ дуг, помеченных числами от 1 до $m$. Концы дуг обозначим через $\beta_{1}, \ldots, \beta_{m}$. Пусть $V_{i}=V \cap X_{i}, i=1, \ldots, m$.

Для каждого $i \in\{1, \ldots, m\}$ выпустим из $\beta_{i} 2-3$ дерево с библиотекой $V_{i}$. Очевидно, что полученный информационный граф будет решать задачу поиска.

Операции поиска, вставки и удаления записи в построенном дереве совершаются по следующему алгоритму: вначале вычисляется значение переключателя $g_{m}(x)$ на запросе, а затем поиск, вставка или удаление производится в соответствующем 2-3 поддереве. Обозначим $A_{1}$ такой алгоритм вставки/удаления записи в 2-3 дереве.

Предложение 4. Пусть $U \in \mathcal{U}\left(A_{1}, n\right)-$ ИГ с п листьями, построенный по алгоритму $A_{1}$. тогда

$$
\begin{array}{lll}
T(U) \leqslant t_{g}+\frac{n}{m} t_{\lambda}, & \widehat{T}(U) \leqslant t_{g}+t_{\lambda}\left\lfloor\log _{2} n\right\rfloor, \\
R(U) \leqslant t_{g}+\frac{n}{m}\left(t_{\lambda}+t_{2}\right), & & \widehat{R}(U) \leqslant t_{g}+\left(t_{\lambda}+t_{2}\right)\left\lfloor\log _{2} n\right\rfloor, \\
S(U) \leqslant t_{g}+\frac{n}{m}\left(t_{\lambda}+t_{2}+t_{3}\right), & & \widehat{S}(U) \leqslant t_{g}+\left(t_{\lambda}+t_{2}+t_{3}\right)\left\lfloor\log _{2} n\right\rfloor .
\end{array}
$$

Доказательство. Пусть $x$ - произвольный запрос из $X$. Пусть, далее, $i$ - номер подотрезка $X_{i}$, в который попадает $x$. Обозначим через $l_{i}$ мощность множества $V_{i}, i=1, \ldots, m$. Тогда, применив предложение 2 , получим для сложности поиска, вставки и удаления записи $x$ в ИГ $U$ оценки

$$
\begin{aligned}
& T(U, x) \leqslant t_{g}+t_{\lambda}\left\lfloor\log _{2} l_{i}\right\rfloor, \\
& R(U, x) \leqslant t_{g}+\left(t_{\lambda}+t_{2}\right)\left\lfloor\log _{2} l_{i}\right\rfloor, \\
& S(U, x) \leqslant t_{g}+\left(t_{\lambda}+t_{2}+t_{3}\right)\left\lfloor\log _{2} l_{i}\right\rfloor .
\end{aligned}
$$

Из этих неравенств, а также очевидных соотношений $l_{i} \leqslant n, i \in\{1, \ldots, m\}$, получим искомые оценки верхней сложности основных операций.

Оценим теперь величины соответствующих сложностей в среднем. Нетрудно видеть, что

$$
\begin{aligned}
T(U) & =\mathbf{M}_{x}(T(U, x))=\int_{X} T(U, x) \mathbf{P}(d x)=\sum_{i=1}^{m} \int_{X_{i}} T(U, x) \mathbf{P}(d x) \\
& \leqslant \sum_{i=1}^{m} \int_{X_{i}}\left(t_{g}+t_{\lambda}\left\lfloor\log _{2} l_{i}\right\rfloor\right) \mathbf{P}(d x)=t_{g}+t_{\lambda} \sum_{i=1}^{m}\left\lfloor\log _{2} l_{i}\right\rfloor \mathbf{P}\left(X_{i}\right) \\
& \leqslant t_{g}+t_{\lambda} \sum_{i=1}^{m} \frac{l_{i}}{m}=t_{g}+\frac{n}{m} t_{\lambda} .
\end{aligned}
$$

Аналогичным образом получим, что

$$
R(U) \leqslant t_{g}+\frac{n}{m}\left(t_{\lambda}+t_{2}\right), \quad S(U) \leqslant \frac{n}{m}\left(t_{\lambda}+t_{2}+t_{3}\right) .
$$


Замечание 1. В случае, если в описываемом ИГ $U$, в котором $N(U)=n$, выполняется неравенство $n \leqslant c m$, где $c-$ некоторая постоянная, то средняя сложность основных операций по величине не больше, чем константа, а именно,

$$
\begin{aligned}
& T(U) \leqslant t_{g}+\frac{n}{m} t_{f} \leqslant t_{g}+c t_{f}, \\
& R(U) \leqslant t_{g}+\frac{n}{m} t_{f}+t_{\alpha} \leqslant t_{g}+c t_{f}+t_{\alpha}, \\
& S(U) \leqslant t_{g}+\frac{n}{m} t_{f}+t_{\beta} \leqslant t_{g}+c t_{f}+t_{\beta} .
\end{aligned}
$$

Из замечания 1 следует, что для того чтобы основные операции сохраняли константную сложность, можно по мере роста числа записей в БД увеличивать значение параметра хеш-функции $m$ и соответствующим образом перестраивать ИГ $U$. При этом процедура, выполняющая преобразование $U$, не должна тормозить эти основные операции.

Будем считать, что операция перестройки ИГ выполняется независимо от операций поиска и вставки в ИГ и параллельно им. Это значит, что параллельно с основным процессом, выполняющим поиск и вставку записей, работает второй процесс, периодически совершающий перестройку ИГ. Этот служебный процесс будет блокировать доступ к какой-то части вершин, пока он перестраивает их. Тогда параллельный ему основной процесс, дойдя до заблокированной вершины, будет вынужден ждать ее разблокировки. Таким образом, сложность поиска и вставки записи (которая понимается нами как время выполнения некой абстрактной процедуры) может увеличиться.

Итак, для достижения константной верхней оценки сложности основных операций необходимо, чтобы

(1) процедура перестройки, меняющая значение параметра хеш-функции $m$ на $m^{\prime}$, заканчивалась до того, как число записей в БД достигало значения $\mathrm{cm}^{\prime}$, где величина $c$ постоянна на протяжении всего функционирования БД,

(2) увеличение средней сложности основных операций (с учетом возможной блокировки обрабатываемых ими вершин) произошло не более, чем на константу.

Пусть перед началом этой перестройки переменная $m$ имела значение $m_{0}$. В результате перестройки ИГ эта переменная будет принимать значение $m_{1}=2 m_{0}$.

Рассмотрим новое разбиение полуотрезка $X=(0,1]$, а именно, разбиение

$$
\mathscr{X}^{\prime}=\left\{X_{1}^{\prime}, \ldots, X_{m_{1}}^{\prime}\right\}, \quad X_{i}^{\prime}=\left(\frac{i-1}{m_{1}}, \frac{i}{m_{1}}\right], \quad i=1, \ldots, m_{1} .
$$

Для каждой из вершин $\beta_{i}$ произведем следующее.

(1) Заблокируем для основного процесса вершину $\beta_{i}$.

(2) Отцепим 2-3 поддерево $U_{i}$ от основного ИГ $U$.

(3) Произведем операцию деления $U_{i}$ пополам. Обозначим полученные деревья $U_{2 i-1}^{\prime}$ и $U_{2 i}^{\prime}$.

(4) Выпустим из $\beta_{i}$ две 2 дуги, помеченные числами $2 i-1$ и $2 i$, и навесим на каждую из них соответствующее из деревьев $U_{2 i-1}^{\prime}$ и $U_{2 i}^{\prime}$. Корни 2-3 деревьев обозначим через $\beta_{2 i-1}^{\prime}$ и $\beta_{2 i}^{\prime}$. Сопоставим вершине $\beta_{i}$ переключатель $g_{m_{1}}(x)$. Будем считать, что сложность такой операции равна $2 t_{\gamma}$. 
(5) Разблокируем $\beta_{i}$.

Из описания алгоритма и предложения 3 получим верхнюю оценку времени выполнения перестройки $i$-го поддерева $2 t_{\gamma}+\left(t_{\lambda}+2 t_{2}+2 t_{3}+t_{4}\right)\left\lfloor\log _{2} l_{i}^{\prime}\right\rfloor$, где $l_{i}^{\prime}-$ количество записей в поддереве $U_{i}$ в момент начала его перестройки.

Когда описанная процедура выполнена для всех вершин $\beta_{i}$, склеим все вершины $\beta_{i}$, а полученную вершину назначим корнем основного дерева (оставив приписанный ей переключатель $\left.g_{m_{1}}(x)\right)$. Мы можем считать эту операцию элементарной и выполняемой мгновенно. Заметим, что такое предположение основано на том, что в практической реализации предложенной модели набору вершин $\beta_{i}$ и набору вершин $\beta_{j}^{\prime}$ логично сопоставить массив указателей на поддеревья, тогда операция склейки заключается всего лишь в замене хеш-функции $g_{m_{0}}(x)$ на $g_{m_{1}}(x)$ и замене в ней адреса начала массива.

Таким образом, вся перестройка ИГ $U$ займет времени не больше, чем

$$
\begin{aligned}
\sum_{i=1}^{m_{0}}\left(2 t_{\gamma}+\left(t_{\lambda}+2 t_{2}+2 t_{3}+t_{4}\right)\left\lfloor\log _{2} l_{i}^{\prime}\right\rfloor\right) & \leqslant 2 m_{0} t_{\lambda}+\left(t_{\lambda}+2 t_{2}+2 t_{3}+t_{4}\right) \sum_{i=1}^{m_{0}} l_{i}^{\prime} \\
& =m_{1} t_{\gamma}+\left(t_{\lambda}+2 t_{2}+2 t_{3}+t_{4}\right) N^{\prime}(U),
\end{aligned}
$$

где $N^{\prime}(U)$ - то, каким было бы число записей в ИГ $U$ к концу выполнения перестройки, если во время этой перестройки записи не удалялись.

Исследуем, как изменится время поиска, вставки и удаления во время перестройки. Каждая из этих операций будет состоять из следующих этапов

(1) Вычисление переключателя $g_{m_{0}}(x)$, приписанного корню.

(2) Поиск, вставка или удаление в поддереве с номером $i=g_{m_{0}}(x)$. Здесь возможны следующие варианты:

(a) служебный процесс не дошел до поддерева с номером $i$, тогда сложность любой операции не изменяется,

(б) служебный процесс обрабатывает текущее поддерево, тогда основной процесс блокируется до окончания преобразования, а затем производится поиск (вставка, удаление) записи $x$ в новом поддереве $U_{i}^{\prime}$,

(в) служебный процесс обработал поддерево $U_{i}$, тогда соответствующая операция совершается в поддереве $U_{i}$.

Обозначим $A_{2}$ измененный алгоритм выполнения основных операций. Сформулируем новое утверждение, касающееся сложности основных операций в построенной модели.

Предложение 5. Для ИГ $U$ такого, что $U \in U\left(A_{2}, n\right)$ справедливы оценки

$$
\begin{aligned}
& \widehat{T}(U) \leqslant 2 t_{g}+2 t_{\gamma}+\left(2 t_{\lambda}+2 t_{2}+2 t_{3}+t_{4}\right)\left\lfloor\log _{2} n\right\rfloor ; \\
& \widehat{R}(U) \leqslant 2 t_{g}+2 t_{\gamma}+\left(2 t_{\lambda}+3 t_{2}+2 t_{3}+t_{4}\right)\left\lfloor\log _{2} n\right\rfloor ; \\
& \widehat{S}(U) \leqslant 2 t_{g}+2 t_{\gamma}+\left(2 t_{\lambda}+3 t_{2}+3 t_{3}+t_{4}\right)\left\lfloor\log _{2} n\right\rfloor ; \\
& T(U) \leqslant 2 t_{g}+2 t_{\gamma}+\left(2 t_{\lambda}+2 t_{2}+2 t_{3}+t_{4}\right) \frac{n}{m} \\
& R(U) \leqslant 2 t_{g}+2 t_{\gamma}+\left(2 t_{\lambda}+3 t_{2}+2 t_{3}+t_{4}\right) \frac{n}{m} \\
& S(U) \leqslant 2 t_{g}+2 t_{\gamma}+\left(2 t_{\lambda}+3 t_{2}+3 t_{3}+t_{4}\right) \frac{n}{m}
\end{aligned}
$$


Доказательство. Пусть $x$ - произвольный запрос к БД, а $i=g_{m}(x)$. Из рассуждений, проведенных выше получим, что

$$
\begin{aligned}
T(U, x) & \leqslant 2 t_{g}+2 t_{\gamma}+\left(t_{\lambda}+2 t_{2}+2 t_{3}+t_{4}\right)\left\lfloor\log _{2} l_{i}\right\rfloor+t_{\lambda}\left\lfloor\log _{2} l_{i}\right\rfloor \\
& \leqslant 2 t_{g}+2 t_{\gamma}+\left(2 t_{\lambda}+2 t_{2}+2 t_{3}+t_{4}\right)\left\lfloor\log _{2} l_{i}\right\rfloor ; \\
R(U, x) & \leqslant 2 t_{g}+2 t_{\gamma}+\left(t_{\lambda}+2 t_{2}+2 t_{3}+t_{4}\right)\left\lfloor\log _{2} l_{i}\right\rfloor+\left(t_{\lambda}+t_{2}\right)\left\lfloor\log _{2} l_{i}\right\rfloor \\
& \leqslant 2 t_{g}+2 t_{\gamma}+\left(2 t_{\lambda}+3 t_{2}+2 t_{3}+t_{4}\right)\left\lfloor\log _{2} l_{i}\right\rfloor ; \\
S(U, x) & \leqslant 2 t_{g}+2 t_{\gamma}+\left(t_{\lambda}+2 t_{2}+2 t_{3}+t_{4}\right)\left\lfloor\log _{2} l_{i}\right\rfloor+\left(t_{\lambda}+t_{2}+t_{3}\right)\left\lfloor\log _{2} l_{i}\right\rfloor \\
& \leqslant 2 t_{g}+2 t_{\gamma}+\left(2 t_{\lambda}+3 t_{2}+3 t_{3}+t_{4}\right)\left\lfloor\log _{2} l_{i}\right\rfloor .
\end{aligned}
$$

Из этих неравенств следуют оценки верхней сложности основных операций.

Оценка для средней сложности основных операций получается с помощью выкладок, аналогичных проведенных в доказательстве предложения 4:

$$
\begin{aligned}
T(U) & =\mathbf{M}_{x} T(U, x)=\int_{X} T(U, x) \mathbf{P}(d x)=\sum_{i=1}^{m} \int_{X_{i}} T(U, x) \mathbf{P}(d x) \\
& \leqslant \sum_{i=1}^{m}\left(2 t_{g}+2 t_{\gamma}+\left(2 t_{\lambda}+3 t_{2}+2 t_{3}+t_{4}\right)\left\lfloor\log _{2} l_{i}\right\rfloor\right) \mathbf{P}\left(X_{i}\right) \\
& =2 t_{g}+2 t_{\gamma}+\left(2 t_{\lambda}+3 t_{2}+2 t_{3}+t_{4}\right) \sum_{i=1}^{m} \frac{l_{i}}{m} \\
& \leqslant 2 t_{g}+2 t_{\gamma}+\left(2 t_{\lambda}+3 t_{2}+2 t_{3}+t_{4}\right) \frac{n}{m} .
\end{aligned}
$$

Остальные неравенства доказываются аналогично.

Замечание 2. Вообще говоря, в рассуждениях о средней сложности поиска, вставки и удаления записи можно также заметить, что вероятность попасть в поддерево, которое перестраивается, не превышает величины $1 / m$, а это в свою очередь означает, что реальная средняя сложность ощутимо меньше полученных оценок.

Теорема 1. Пусть операция вставки в построенной БД выполнялась не чаще, чем один раз за время $2\left(t_{\gamma}+t_{\lambda}+2 t_{2}+2 t_{3}+t_{4}\right)$. Пусть также перестройка в ИГ U совершается как только число записей в БД достигает $m$. Тогда

$$
\begin{aligned}
& T(U) \leqslant 2 t_{g}+2 t_{\gamma}+2\left(2 t_{\lambda}+2 t_{2}+2 t_{3}+t_{4}\right), \\
& R(U) \leqslant 2 t_{g}+2 t_{\gamma}+2\left(2 t_{\lambda}+3 t_{2}+2 t_{3}+t_{4}\right), \\
& S(U) \leqslant 2 t_{g}+2 t_{\gamma}+2\left(2 t_{\lambda}+3 t_{2}+3 t_{3}+t_{4}\right) .
\end{aligned}
$$

Доказательство. Ранее было показано, что перестройка ИГ $U$ занимает время

$$
t \leqslant m_{1} t_{\gamma}+\left(t_{\lambda}+2 t_{2}+2 t_{3}+t_{4}\right) n
$$

где $n$ - суммарное число листьев в поддеревьях $U_{i}^{\prime}$ на момент обработки соответствующего поддерева.

С другой стороны, даже если вставки идут максимально часто, время, необходимое для того, чтобы число обработанных вершин достигло $n$, удовлетворяет неравенству

$$
t \geqslant 2\left(t_{\gamma}+t_{\lambda}+2 t_{2}+2 t_{3}+t_{4}\right)\left(n-n_{0}\right),
$$


где $n_{0}-$ число записей в БД перед началом перестройки. Получаем неравенство

$$
2\left(t_{\gamma}+t_{\lambda}+2 t_{2}+2 t_{3}+t_{4}\right)\left(n-n_{0}\right) \leqslant m_{1} t_{\gamma}+\left(t_{\lambda}+2 t_{2}+2 t_{3}+t_{4}\right) n .
$$

Напомним, что перестройка начинается в момент, когда число записей достигает $m$, значит $n_{0}=m_{0}$. Получаем неравенство

$$
n \leqslant 2 n_{0} \frac{2 t_{\gamma}+t_{\gamma}+t_{2}+2 t_{3}+t_{4}+\varepsilon}{2 t_{\gamma}+t_{\gamma}+t_{2}+2 t_{3}+t_{4}+\varepsilon}=2 n_{0},
$$

а это значит, что перестройка заканчивается до того, как число записей в БД достигает значения $2 n_{0}$ и нужно совершать очередную перестройку. Более того, в каждый момент времени выполняется неравенство $N^{\prime}(U) \leqslant 2 m$. Тогда, пользуясь предложением 5 , получаем искомые оценки.

Итак, мы получили такую структуру БД, которая позволяет выполнять все основные операции за константное в среднем время. С другой стороны, верхняя оценка сложности имеет логарифмический порядок, такой же, как у большинства древовидных структур, использующихся для реализации динамических баз данных.

\section{Список литературы}

1. Лапшов И. С., Динамические базы данных, основывающиеся на хешировании методом цепочек. Интеллектуальные системы (2005) 9, №1/4, 191-207.

2. Лапшов И. С., О динамических базах данных с константной в среднем сложностью поиска и вставки. В сб.: Тез. докл. ХIV Международной конф. «Проблемы теоретической кибернетики». Изд-во мех.-матем. ф-та МГУ, Москва, 2005, с. 85.

3. Гасанов Э. Э., Кудрявцев В. Б., Теория хранения и поиска информачии. Физматлит, Москва, 2002.

4. Ахо А., Хопкрофт Дж., Ульман Дж., Построение и анализ вычислительных алгоритмов. Мир, Москва, 1979.

5. Dumey A., Indexing for rapid random access memory systems. Computers and Automation (1956) 4, №12, 6-9.

6. Кнут Д., Искусство программирования для ЭВМ, 3. Мир, Москва, 1978.

Статья поступила 10.07.2006. 